\title{
Mechanical stiffening of human rhinovirus by cavity-filling antiviral drugs
}

Alejandro Valbuena, Alicia Rodríguez-Huete, and Mauricio G. Mateu*

Centro de Biología Molecular "Severo Ochoa" (CSIC-UAM), Universidad Autónoma de Madrid, Madrid 28049, Spain

*Correspondence to M.G.M.: mgarcia@cbm.csic.es

Running title: Rhinovirus stiffening by antiviral drugs 
Emerging studies at the nanoscale on relationships between structure, mechanical properties and infectivity of virus particles are revealing important physics-based foundations of virus biology that may have biomedical and nanotechnological applications. Human rhinoviruses (HRV) are the major causative agents of common colds leading to important economic losses, and are also associated with more severe diseases. There is a renewed interest in developing effective anti-HRV drugs, but none has been approved so far. We have chosen HRV to explore a possible link between virus mechanics and infectivity and the antiviral effect of certain drugs. In particular, we have investigated the suggestion that the antiviral action of drugs that bind into capsid cavities (pockets) may be related to changes in virus stiffness. Mechanical analysis using atomic force microscopy shows that filling the pockets with drugs or genetically introducing bulkier amino acid side chains in the pockets stiffen HRV virions to different extents. Drug-mediated stiffening affected regions distant from the pockets and involved in genome uncoating, and may be caused by a subtle structural rearrangement of the virus particle. The results also revealed for HRV a quantitative, logarithmic relationship between mechanical stiffening, achieved either by drug binding or introducing bulkier amino acid side chains in the pockets, and reduced infectivity. From a fundamental physics perspective, these drugs may exert their biological effect by decreasing the deformability of the virion, thus impairing its equilibrium dynamics. The results encourage the design of novel antiviral drugs that inhibit infection by mechanically stiffening the viral particle. 


\section{Introduction}

Viruses are increasingly being studied and understood from the perspective of fundamental physics at the nanoscale (reviewed in ${ }^{1-14}$ ). Recent analyses using single-molecule techniques, especially atomic force microscopy (AFM), ${ }^{4,6,8,12,15-31}$ and/or molecular dynamics (MD) simulations $^{4,9,10,32-35}$ are revealing that different spherical viruses show quite diverse responses when subjected to mechanical forces. Stiffness, brittleness, strength against disruption by point forces and/or material fatigue have been compared between different virus particles. ${ }^{6}$ Growing experimental evidence, including our studies with a structurally very simple virus (the minute virus of mice, $\mathrm{MVM}^{36}$ ), suggests that the diverse mechanical behavior of viruses may have genetically arisen in response to different natural selection pressures to enable or optimize important biological functions: resistance to disruption or inactivation by physicochemical agents, entry into or exit from cells, assembly, maturation or translocation, genome encapsidation or ejection, etc. ${ }^{2-6,8,12,13,16,18,22,25-31}$

In this study we have chosen human rhinovirus (HRV) $)^{37,38}$ to explore if a connection exists between virus mechanics, and infectivity and their inhibition by some antiviral drugs. More specifically, we have investigated the suggestion that the antiviral action of certain drugs may be fundamentally related to changes in mechanical stiffness of the virus particle.

HRV belongs to the enterovirus genus within the Picornaviridae family. ${ }^{37}$ Picornaviruses are the most common viral pathogens, and HRVs are the major causative agents of common colds, which lead to millions of lost working and school days and important economic losses each year. ${ }^{39}$ During the last decades, a great deal of knowledge has been obtained on the structural basis and molecular mechanisms of infection by HRV and other enteroviruses, the action of anti-enteroviral drugs, and the mechanisms of drug resistance by enteroviruses (reviewed in ${ }^{37,40-45}$ ). In recent years, new developments have led to a general awareness that HRVs (including a recently discovered species, HRV-C) ${ }^{46}$ are associated with more severe diseases, such as exacerbation of asthma and chronic obstructive pulmonary disease (COPD) ${ }^{39}$ The World Health Organization has predicted that COPD could become the third leading cause of death worldwide by 2030. These and other observations have elicited renewed, intense interest in developing effective drugs against HRV ${ }^{47-49}$ as exemplified by the many research articles on novel anti-HRV compounds published in the last $\sim 5$ years. Past clinical trials with a few drug candidates, including pleconaril, led to moderately promising 
results, ${ }^{49,50}$ and other trials are in progress. However, no drug against infection by HRV (or any other enterovirus) has been approved so far.

HRVs (and all picornaviruses) are composed of a ssRNA genome enclosed in a $\mathrm{T}=1$ $(\mathrm{P}=3)$ icosahedral capsid. ${ }^{38}$ The capsid $(d=30 \mathrm{~nm})$ is made of 60 identical hetero-oligomeric protomers, each formed by one copy of 3 proteins (VP1, VP2, VP3) and an internal polypeptide (VP4) (Figs. 1A,B). A characteristic feature of HRVs (and other enteroviruses) is the canyon, a large depression that surrounds each capsid 5-fold symmetry (S5) axis (Figs. 1A,B) ${ }^{38,40}$ In most HRVs (and other enteroviruses), a hydrophobic cavity or pocket under the canyon is accessible to small molecules through an opening at the base of the canyon (Fig. 1C). ${ }^{38,40}$

Major receptor group HRVs (including HRV-B14 (HRV14) used in this study), polioviruses and other enteroviruses start infection by binding receptor proteins on the host cell membrane. The cell receptor (ICAM-1 for major receptor group HRVs) binds the metastable virion (160S particle) by penetrating deep into the canyon. Receptor binding triggers a structural rearrangement of the viral particle required for genome uncoating and replication (reviewed in ${ }^{41,42,44,45}$ ). This rearrangement proceeds stepwise through two major conformational transitions that must overcome large activation free energy barriers. Receptor binding facilitates a first transition towards a structurally rearranged viral particle (135S) in which VP4 has been released and the VP1 N-terminus (Nt) is permanently externalized. ${ }^{42,44}$ Afterwards, a second transition occurs that involves release of the viral RNA through a larger opening, leaving an empty capsid (80S). ${ }^{42,44}$

A large number of elongated, hydrophobic organic compounds efficiently inhibit infection by many enteroviruses. ${ }^{47-51}$ These compounds exert their antiviral effect by binding inside the capsid pockets in HRV (Figs. 1B,C), ${ }^{40,41,43,52-55}$ poliovirus and/or other enteroviruses. Solution studies using techniques such as limited proteolysis-mass spectrometry (LP-MS) have shown that HRV and poliovirus are conformationally dynamic at equilibrium, transiently exposing parts of capsid proteins VP1 and VP4 through capsid openings. ${ }^{56-62}$ This "breathing" 58 of the viral particle is severely impaired by pocket-binding antiviral drugs. ${ }^{58,59,61,62}$ It has been proposed that, in the absence of drug (empty pockets), virus breathing allows the frequent adoption of an "open" virus conformation from a basal, "closed" conformation; the cellular receptor specifically binds this open conformation with highaffinity and triggers the structural rearrangements leading to infection. ${ }^{42,44,45,58,59,61,62,63}$ Drug binding into the pockets reduces the probability to adopt the "open" conformation, thus 
impairing receptor binding and the receptor-mediated structural rearrangement required for infection.

It has been suggested that pocket-binding drugs, by nearly completely filling the capsid pockets, could fundamentally exert their action by increasing the rigidity of the virion, which would impair virus breathing, receptor binding and the conformational transitions required for genome uncoating. ${ }^{53,54,63}$ However, the mechanical properties of HRV or any other picornavirus, the putative change in mechanical stiffness caused by antiviral drugs or mutation, and any relationship of any stiffening effect with impaired breathing and/or loss of infectivity, had not been experimentally investigated so far.

Here we have used AFM to analyze and quantify, in close to physiological conditions, the mechanical stiffness of the HRV14 virion in the absence or presence of pocket-binding antiviral drugs, and of HRV14 variants carrying mutations in the capsid pockets. The different mechanical behavior experimentally determined in each case is discussed in connection with: i) structural changes in the virion; and ii) the biological action of antienteroviral drugs that inhibit infection by impairing uncoating.

\section{Results}

\section{Topographic imaging of HRV14 particles by AFM}

For topographic and mechanical analyses by AFM of HRV14 virions in liquid we used purified virus suspensions that were diluted enough to obtain well-separated particles adsorbed onto a solid substrate. Based on previous experiences on imaging $\mathrm{HRV}^{64}$ and other virus particles, ${ }^{15,16,65}$ using AFM, various substrates (including glass, silanized glass and freshly cleaved mica) and buffer solutions (Tris buffer at neutral $\mathrm{pH}$ containing $\mathrm{Ni}^{2+}$ ions ${ }^{64}$ and phosphate-buffered saline, PBS) were tested. With any of those substrates and buffers, and using the cantilevers we had extensively used for AFM analysis of other viruses, ${ }^{23}$ we encountered three difficulties for regular high-resolution imaging and mechanical analysis of HRV virions: i) non-specific force peaks appeared with some frequency due to adhesion between particle and cantilever tip; ii) some particles were disrupted during small-field scanning (Fig. S1 of Supplementary Information, SI), suggesting weak mechanical strength compared to other viruses (e.g., $\mathrm{MVM}^{16}$ ); iii) many particles were displaced by the tip, even when exerting very low forces $(\sim 10-80 \mathrm{pN})$ and using Jumping Mode ${ }^{66}$ to minimize both particle damage and positional shifts, suggesting weak adsorption. These problems were 
minimized by improving the AFM setup, mainly using a somewhat softer cantilever (see Experimental), which allowed high-resolution imaging and mechanical analysis of isolated HRV14 virions. As no significant differences were observed when different substrates and buffers were used, we carried out all subsequent AFM imaging and mechanical analysis using silanized glass as a substrate and PBS as a physiological buffer.

Virion height $h$ was determined at nanometric resolution $(h=30.2 \pm 0.6 \mathrm{~nm}$ in PBS), and was indistinguishable (within error) from the HRV14 diameter obtained by X-ray crystallography $^{38}\left(d=31.4 \mathrm{~nm}\right.$ as given in VIPERdb $\left.{ }^{67}\right)$. High-resolution imaging allowed us to determine the orientation of individual virus particles by identifying major topographic features (protrusions and depressions) and the icosahedral symmetry axis (S5, S3 or S2) located close to the top of the particle (Fig. 2). Please compare a HRV14 atomic model shown with a S2, S3 or S5 axis on top (Fig. 2, left) with actual AFM images of particles in approximately the same orientations (Fig. 2, center left). Plotting in different colors the contour lines corresponding to different heights revealed upper contours that define approximately the shape of a rhombus ( 2 2 symmetry, top), a triangle (S3 symmetry, center) or a pentagon (S5 symmetry, bottom). To provide further objective confirmation for assignment of individual particles according to their orientation, we used a tool implemented in the WSxM software called Flooding. This tool is able to "cut" the viral particle image at a determined height, which enabled us to plot only the contour corresponding to a defined height, close to the top of the particle (Fig. 2, center right). These filtered images were fed into a software that is able to identify polygons of different shapes. The polygons selected by the software corresponded, as expected, to a rhombus (for particles that had been assigned to the group with a S2 axis on top), a triangle (for particles assigned to the group with a S3 axis on top) or a pentamer (for particles assigned to the group with a S5 axis on top) (Fig. 2, right).

To sum up, AFM imaging of isolated HRV14 virions in physiological buffer showed that: i) substrate-bound virions are weakly adsorbed, generally keep their integrity and are not deformed; ii) virions can be adsorbed in any orientation, which can be unambiguously ascertained in high-resolution images.

\section{The HRV14 virion is isotropic regarding mechanical stiffness}

To determine the mechanical stiffness of the HRV14 virion in a physiological buffer (PBS), nanoindentations were performed by pushing with the AFM tip centered on isolated virions, to obtain force-z displacement $(F-Z)$ curves. $^{15,16}$ The viral particle was kept under the elastic regime by applying indentations smaller than $2 \mathrm{~nm}$, as determined from converted force- 
particle deformation (indentation) $(F-D)$ curves. ${ }^{16,19}$ Representative force curves are shown in Fig. S2 of SI. The regions corresponding to the elastic regime were fitted to straight lines. Fittings were generally excellent with regression values very close to one; differences in slope between traces obtained either by indenting the hard substrate or the soft viral particles were clearly and reproducibly observed. Elastic constant $\left(k_{e}\right)$ values were determined as indicated in the Experimental section. ${ }^{15,16}$ All experiments on HRV mechanics were carried out with virions submerged in PBS buffer at neutral $\mathrm{pH}$ and $25^{\circ} \mathrm{C}$.

Indentations on many isolated, intact HRV14 virions were carried out to obtain a statistically significant value for their overall (average) stiffness. A normal, quite narrow unimodal distribution of $k_{e}$ values was obtained (average \pm standard deviation $k_{e}=0.49 \pm 0.20$ $\mathrm{N} / \mathrm{m}$ ) (Fig. 3 and Table 1). The very low dispersion of $k_{e}$ values suggests that, under these conditions, the virion is nearly isotropic regarding stiffness. This suggestion was confirmed by determination of $k_{e}$ values for different regions of the HRV14 virion (those centered around S2, S3 or S5 axes) by repeated indentations of a number of particles for which orientation was unambiguously determined as described above (Fig. 4, WT, and Table 1).

\section{Pocket-binding antiviral drugs increase the mechanical stiffness of the HRV14 virion}

A fundamental physical effect of filling the HRV capsid pockets with antiviral drug molecules (Figs. 1B,C) could be an increase in capsid rigidity. This effect would be reflected in an increased average $k_{e}$ of the HRV virion as determined by AFM in indentation experiments. To test this possibility, we compared the average stiffness of the HRV14 virion both in the absence or presence of virus-inactivating concentrations of either of two wellknown pocket-binding antiviral drugs, pirodavir and pleconaril. ${ }^{47,48,50}$

The antiviral effect of these drugs in our experimental conditions were first quantified in viral infectivity assays (Fig. 5). A fixed amount of purified HRV14 virions were incubated with increasing drug concentrations, and then used to infect susceptible cell monolayers. After appropriate times post-infection, infectious progeny viruses were collected and titrated (see Experimental). The relative antiviral action of both pirodavir (Fig. 5A) and pleconaril (Fig. 5B) detected in these experiments was similar to that previously described for these drugs using other ex vivo assays. ${ }^{47}$ Based on these results, we concluded that a $0.5 \mu \mathrm{M}$ concentration of either drug is enough to cause a drastic ( $\sim 99 \%-99.9 \%)$ inhibition of HRV14 infectivity in our assays. Previous structural analysis indicates that, under these conditions, most (albeit not all) of the 60 identical capsid pockets in the HRV capsid are occupied by a drug molecule. ${ }^{53}$ 
Then, we incubated purified HRV14 virions with $0.5 \mu \mathrm{M}$ pirodavir or pleconaril in PBS, and carried out a series of indentation experiments in the same conditions used to determine $k_{e}$ for the untreated HRV14 virion (Fig. 6). Relative to the average intrinsic stiffness of HRV14 in the absence of drug, binding of either drug to the capsid pocket led to significant, drastic increases in average stiffness (Fig. 6, Table 1, and Table S1 of SI). At the same concentration, both drugs caused a substantial stiffening (by $75 \%$ with pirodavir, $73 \%$ with pleconaril). Drug binding led also to a higher dispersion of $k_{e}$ values. This observation suggested the possibility that the drug-mediated stiffening could be anisotropic.

The mechanical stiffening of the HRV14 virion induced by a pocket-binding drug is anisotropic

We then analyzed whether the observed drug-induced stiffening of the HRV14 virion is isotropic or anisotropic, and which particular regions of the viral particle are stiffened by pocket-bound drugs. The orientation on the substrate was ascertained for a number of isolated, intact HRV14 virions with bound pirodavir or pleconaril, and local $k_{e}$ values for S2, S3 or S5 regions were estimated. While drug-free HRV14 is mechanically isotropic regarding stiffness, virions bound to pirodavir or pleconaril were mechanically highly anisotropic (compare Fig. 4, Table 1, and Table S2 of SI). Drug binding dramatically stiffened S2 and S3 regions, but it did not significantly stiffen S5 regions.

\section{Drug binding into the pockets of the HRV14 virion may cause changes in mobility and conformation of distant structural elements, and a subtle structural compaction of the viral particle}

The above results revealed that pocket-binding drugs stiffen the HRV14 virion at S2 and S3 regions, which are quite distant from the bound drug (by $\sim 37 \AA$ or $\sim 63 \AA$, respectively; see Fig. 1B). To try and understand the structural basis for this stiffening, we looked for effects of pocket-binding drugs on the precise conformation and mobility of structural elements, especially those distant from the capsid pockets.

As an indicator of possible changes in atomic mobility for each capsid residue, B-factors obtained for the corresponding $\mathrm{C} \alpha$ atom in the X-ray structures of the HRV14 virion, without or with bound drug, were normalized (to get $\mathrm{B}_{\text {norm }}$ values) as described in Experimental, and compared (pleconaril only, as no HRV14-pirodavir complex structure is available). The results (Fig. S3 of SI and Fig. 7) revealed that, in the presence of drug, $B_{\text {norm }}$ values significantly increased for 74 capsid residues and decreased for 79 other residues. However, 
reductions in $\mathrm{B}_{\text {norm }}$ values were generally larger (by about twofold) than increases in $\mathrm{B}_{\text {norm }}$ values (Fig. S3 of SI). Remarkably, in the HRV-drug complex the regions surrounding the drug-filled pockets include residues with increased $B_{\text {norm }}$ but fewer residues with decreased $\mathrm{B}_{\text {norm }}$; in contrast, capsid regions along the interfaces between pentameric subunits, especially around the $\mathrm{S} 2$ axes, include residues with reduced $\mathrm{B}_{\text {norm }}$, but residues with increased $\mathrm{B}_{\text {norm }}$ are rare (Fig. 7C). The above observations suggest that local mechanical stiffening of some regions (especially S2 regions) in the HRV14 virion upon drug binding may also be reflected in a reduction in mobility of several amino acid residues located in those regions.

In published comparative descriptions of the crystal structure of HRV14 in the absence or presence of pleconaril or other pocket-binding drugs, ${ }^{52,53,55}$ the authors focused mainly in relatively large conformational changes that occur at or around the capsid pockets upon drug binding. However, analysis of root mean square deviations (rmsd) between equivalent atoms in the HRV14 structure without or with bound pleconaril (or other drugs) suggest that more subtle structural differences in the equilibrium position of many atoms may occur in many other regions in the virus particle. These differences may include a slight overall compaction of the HRV particle: according to the VIPER database ${ }^{67}$ the maximum, average (as defined in $\mathrm{VIPER}^{67}$ ) and minimum capsid diameters are 32.2, 31.4 and $21.6 \AA$ for the unliganded HRV14 virion, and 31.8, 31.0 and $21.2 \AA$ for the HRV14 virion with bound pleconaril. This drug-induced, subtle compaction of HRV14 can be detected visually by comparing the structure of a capsid protomer in the absence or presence of the drug (Video S1 of SI).

No direct structural comparison was possible in the case of pirodavir, as no structural model for the HRV14-pirodavir complex is available yet. However, structural comparisons of HRV14 complexed with any of several pocket-binding drugs revealed that binding of any of these drugs, despite their chemical and steric differences, exert conformational effects in the virion that are very similar to those exerted by pleconaril. Thus, it seems reasonable to assume that the same could apply to pirodavir. Comparison of the HRV-related poliovirus X-ray structure solved without and with bound pirodavir ${ }^{68}$ also revealed, in addition to conformational changes around the capsid pockets, other subtle structural differences at distant regions in the virion.

\section{Mutations that introduce bulkier residues in the capsid pocket both increase the mechanical stiffness of the HRV14 virion and lead to reduced infectivity}

We reasoned that if filling HRV pockets with drug molecules stiffens the virion (Fig. 1C, top), genetically introducing bulkier amino acid side chains in the cavity could also lead to 
some virion stiffening. For testing this hypothesis we chose four mutations: V1188L, Y1152W and C1199Y which increase the bulkiness of the corresponding side chains inside the cavity (Fig. 1C, bottom); and V1188A, which has the opposite effect. Mutations V1188L and $\mathrm{C} 1199 \mathrm{Y}$ had previously been identified in many drug-resistant HRV14 mutants. ${ }^{69,70}$ Residue 1199 is located at the pocket entrance, residue 1188 is at the pocket floor well within the cavity, and residue 1152 is located at the distal end of the pocket as seen from the entry point (Fig. 1C).

We used site-directed mutagenesis to individually introduce those four mutations in the capsid protein-coding sequence within the HRV14 infectious clone. The corresponding mutant virions, and the nonmutated (wt) HRV14 virion as a control, were produced in exactly the same conditions, by transfecting cells with equal amounts of viral RNAs transcribed from the corresponding infectious clones (see Experimental). Viral progeny titers were obtained in two or three repeated, independent experiments, each one carried out in duplicate. The results showed that mutations that involved minor changes in side chain size caused no (V1188A) or minor (V1188L) reductions in infectivity, whereas larger increases in side chain size (C1199Y, Y1152W) reduced infectivity by $\sim 95 \%-99 \%$.

Then, average $k_{e}$ values for the four HRV14 mutants were determined by AFM in the same conditions used to determine the average $k_{e}$ for the wt HRV14 virion. Mutations that involved minor changes in side chain size (V1188A, V1188L) caused insignificant effects on average stiffness, whereas larger increases in side chain size (C1199Y, Y1152W) led to a moderate stiffening of the viral particles (Fig. 8, Table 1, and Table S1 of SI). We also compared for mutants V1188L, C1199Y and Y1152W the elastic constants of S2, S3 and S5 regions in the virion (Fig. 4, Table 1, and Table S2 of SI). Contrary to that observed for nonmutated HRV14 upon drug binding (Fig. 4), the stiffening effects by mutations C1199Y and Y1152W were (within error) virtually isotropic. Mutations V1188L and C1199Y are known to prevent drug binding to $\mathrm{HRV}^{69,70}$ and, accordingly, similar elastic constants were observed for these mutants either in the absence or presence of drug (results not shown).

\section{A quantitative, logarithmic linkage between virion mechanical stiffening and reduced infectivity}

To assess whether a quantitative linkage exists between HRV stiffening (by either drug binding or mutations in the pocket) and reduced virus infectivity, we plotted relative infectivity versus average spring constant for the wt virion either in the absence or presence of pleconaril or pirodavir, and for the four HRV mutants (Fig. 9). The results revealed a 
remarkable quantitative, nonlinear relationship between stiffening and reduced infectivity: a linear increase in the average elastic constant led to an approximately logarithmic decrease in infectivity (please note that in Fig. 9 infectivity is plotted using a logarithmic scale). Specifically, infectivity was reduced by one order of magnitude for each $\sim 0.16 \mathrm{~N} / \mathrm{m}$ increase in the average $k_{e}$ of the virion, whether achieved by binding drugs to the capsid pockets or by introducing small-to-large amino acid replacements in the pockets.

\section{Discussion}

\section{Drug- or mutation-induced structural changes in HRV14 leading to mechanical stiffening}

The results of nanoindentation experiments carried out in this study show that introduction of molecules or increasing the size of individual side chains in hydrophobic pockets of HRV14 causes an overall mechanical rigidification of the virion. Near-complete filling of most pockets with antiviral drugs (pleconaril or pirodavir) led to very substantial virion stiffening. Introduction in every pocket of bulkier side chains by small-to-large replacements of amino acid residues lining the cavity (C1199Y or Y1152W) caused a moderate stiffening. In contrast, amino acid substitutions that involved only very small decreases or increases in side chain size (V1188A, V1188L) had no significant effect on stiffness.

What structural changes induced by drugs or mutations may cause this overall rigidification of HRV14? Virion stiffening by pocket-filling drugs could be intuitively compared with globally stiffening a sponge by filling most of its cavities with little stones. In fact, drug binding stiffened S2 and S3 regions in the virion, both of them quite distant from the drug-filled pockets, but it did not stiffen S5 regions, even though these are closer to the pockets (Fig. 1C).

Drug-mediated stiffening of distant regions in the virion may be a consequence of the propagation of induced structural changes: upon binding of different drugs (including pleconaril) to the HRV14 virion (as well as other HRVs), large conformational changes were observed around the pockets; ${ }^{52,53,55}$ but, in addition, these local changes were accompanied by subtle structural differences at distant regions leading to an equally subtle, overall structural compaction of the viral particle (Video S1 of SI).

The proposed connection between subtle but global structural changes, and stiffening of HRV14 is further supported by our previous work on the mechanics of MVM, an unrelated, 
quite different virus. A recent $\operatorname{study}^{71}$ has shown that a single mutation in the MVM capsid leads to propagated structural and mechanical effects similar to those induced by pleconaril binding to HRV14: i) small conformational changes in many structural elements in the viral particle, many of them located far away from the mutated residue; ii) subtle but significant overall structural compaction of the viral particle; iii) drastic stiffening of different regions in the particle, including regions distant from the mutated residue.

The moderate stiffening of the HRV14 virion by mutations C1199Y and Y1152W, which involve increased side chain volume and large changes in side chain stereochemistry could, in principle, be rationalized in terms of: i) altered intracapsid interactions and/or steric constraints; and ii) facilitated propagated conformational changes. While the V1188L mutation, that did not stiffen the virion, introduced just 2 additional van der Waals interactions with neighboring residues, the C1199Y mutation introduced 11 additional van der Waals interactions. The structure of the Y1152W mutant has not been determined, but the side chain (beyond C $\beta$ ) of Y1152 establishes 9 interactions (including one hydrogen bond) with neighboring residues, and the Tyr to Trp mutation may substantially alter this pattern of interactions. Indeed, the crystal structures of both V1188L and C1199Y mutant virions revealed minor conformational changes at the pockets relative to nonmutated HRV14. ${ }^{70}$ Unfortunately additional, subtle structural changes that could be induced in other regions of the virion by these mutations cannot be assessed, as the mutant structures were not refined. However, the observation that mutations $\mathrm{C} 1199 \mathrm{Y}$ and $\mathrm{Y} 1152 \mathrm{~W}$, like pocket-bound drugs, stiffen also virus regions distant from the mutated residue suggests that both mutations, like pocket-binding drugs, may cause subtle propagated structural rearrangements in the viral particle. It must also be considered that the mutations affect all 60 pockets in the virion, which could amplify through cooperativity their effects. In contrast, in virion-drug complexes not all pockets are occupied. Whatever the case, the results reveal that even very small structural differences in HRV may lead to large differences in mechanical behavior.

To sum up: i) modest reductions in capsid pocket size by introducing bulkier amino acid side chains in the absence of substantial conformational rearrangements, are enough to cause significant increases in virus stiffness, even at regions distant from the pockets; ii) nearly complete filling of most pockets by drugs causes not only large local structural changes, but also subtle propagated rearrangements and overall compaction of the virion that can lead to a drastic stiffening of S2 and S3 regions distant from the pockets.

\section{Mechanical stiffness, equilibrium dynamics and infectivity of HRV14}


An approximately logarithmic relationship was observed between mechanical stiffening of HRV14 (caused by either drug binding or small-to-large amino acid replacements in the capsid pockets) and reduced virus infectivity (yield of progeny virions). Specifically, infectivity was reduced by one order of magnitude for each $\sim 0.16 \mathrm{~N} / \mathrm{m}$ increase in the average elastic constant of the virus. The largest stiffening effects and corresponding reductions in infectivity were caused by filling the pockets with drugs. This result provides direct experimental evidence for the original proposal ${ }^{53,54}$ that pocket-binding drugs could fundamentally exert their action by reducing the deformability of the virus particle.

The available evidence suggests that the connection between mechanical stiffening and reduced infectivity in $\mathrm{HRV}$ is mediated by a reduction in the equilibrium dynamics ("breathing") of the virion. Biophysical studies have shown that different viruses in solution are conformationally dynamic at equilibrium. ${ }^{56-62,72-77}$ For HRV14 at physiological temperatures, virion breathing driven by thermal fluctuations is detected by the transient opening of small gaps in the capsid, through which parts of VP4 and VP1 are reversibly externalized. ${ }^{61}$ A current model on the mechanism of genome uncoating in HRV14, other major receptor group HRVs and poliovirus ${ }^{42,44,45,61}$ proposes that, under physiological conditions, unimpaired virion breathing at equilibrium allows the frequent interconversion between a basal (closed) conformation and an (open) conformation to which the cellular receptor specifically binds; receptor binding lowers the free energy barrier of a conformational transition that leads to irreversible externalization of VP4 and VP1 Nts, and ultimately enables exit of the viral RNA from the capsid. In this model, virus breathing at equilibrium keeps the activation free energy barrier of the receptor-mediated conformational transition low enough to allow genome uncoating upon receptor binding. Pocket-binding antiviral drugs (including pleconaril), which severely impair virus breathing ${ }^{59}$ would reduce the probability to adopt the "open" conformation, thus impairing receptor binding and increasing the activation free energy barrier of the receptor-mediated transition required for uncoating.

The results obtained in the present study suggest that the reduced overall deformability of the virion by filling most capsid pockets with drugs impairs its equilibrium dynamics (breathing), thus impairing receptor binding and the associated conformational transition of the virion required for genome uncoating, and drastically reducing virus infectivity. Mutations C1199Y and Y1152W that moderately stiffen the virion are predicted to moderately reduce breathing, thus leading to less substantial reductions in infectivity. Mutations V1188M and C1199W were not deleterious to HRV14 and led to some reduction in virus breathing. ${ }^{61}$ 
However, quantitative equilibrium dynamics measurements for the specific mutants for which elastic constants have been determined (V1188A, V1188L, C1199Y and Y1152W) will be required to assess whether a quantitative linkage does exist in a viral particle between mechanical stiffness and equilibrium dynamics (either globally or locally).

It must be emphasized that both mechanical stiffening and impaired equilibrium dynamics of HRV14 upon drug binding are not restricted to the vicinity of the pockets. In fact, as discussed above, S2 regions distant from the pockets were drastically stiffened by either pleconaril or pirodavir binding. Moreover, drug binding led to small changes in position and mobility of many residues distant from the pockets. Remarkably, MD simulations predicted correlated motions of capsid residues separated by as many as $8.5 \mathrm{~nm}$, and these correlated motions were disrupted in the presence of pocket-binding drugs. ${ }^{78}$ In addition, both pleconaril and pirodavir binding in HRV pockets, like viral DNA binding to the MVM capsid inner wall, ${ }^{29}$ stabilize the virion against thermal inactivation of its infectivity ${ }^{79,80}$ and increase virion stiffness (this study). Taken together, the above observations clearly indicate that pocket-binding drugs and mutations induce linked global changes in virion structure (however subtle), stiffness (resistance to deformation under mechanical force) and equilibrium dynamics (observed as breathing). Virion stiffening may impair not only transitions between different thermodynamic states separated by a high energy barrier (as observed for $\mathrm{MVM}^{23,29}$ ) but also thermal fluctuation-driven transitions between conformations of similar free energy at equilibrium.

It is worth noting in this regard that exit of the RNA molecule during uncoating of both poliovirus and HRV may occur through an opening at one S2 region in the virion. ${ }^{81,82}$ Thus, the antiviral effect of pocket-binding drugs could be related not as much to overall virion stiffening, but to local stiffening of S2 regions.

Mechanical stiffness is a fundamental property of solid objects that, for virus particles, can be readily and precisely quantified in close to physiological conditions by using AFM. By directly probing changes in viral particle stiffness, information could be inferred also on changes in their conformational dynamics. Studies on i) changes in stiffness of different regions in a virus particle (quantified by AFM), ii) changes in accessibility of different regions to proteolysis or hydrogen-deuterium exchange in solution (followed by MS), and iii) changes in atomic mobility (indicated by normalized B factors in X-ray structures, ${ }^{60}$ or predicted by MD simulations ${ }^{78}$ ) may complement and reinforce each other. Together, these approaches may provide deeper insights into the equilibrium dynamics of virus particles, a 
property of critical biological relevance for many viruses, and the relationship between virus mechanics, dynamics and infection.

\section{Experimental}

\section{Recombinant plasmids and site-directed mutagenesis}

The infectious HRV14 clone pWR3.26 was acquired from the American Type Culture Collection (ATCC). This recombinant plasmid was amplified by transformation of E. coli DH5 $\alpha$ and purified. The QuikChange Site-directed Mutagenesis kit (Stratagene) was used to specifically introduce mutations V1188A, V1188L, C1199Y and Y1152W on pWR3.26 essentially as described by the manufacturer. Automated DNA sequencing confirmed the presence of the desired mutation and the absence of other mutations.

\section{In vitro transcription of infectious viral $\mathrm{RNA}$}

For in vitro transcription of the HRV14 (wt and mutants) RNA genome, the corresponding pWR3.26 plasmids were first linearised with restriction endonuclease MluI and purified by phenol extraction. $20 \mathrm{U}$ of T7 RNA polymerase (Promega) were used to transcribe $1 \mu \mathrm{g}$ of purified and linearised DNA in optimized transcription buffer ( $8 \mathrm{mM}$ Tris- $\mathrm{HCl} \mathrm{pH}=7.9,2 \mathrm{mM}$ $\mathrm{NaCl}, 1.2 \mathrm{mM} \mathrm{MgCl} 2,0.4 \mathrm{mM}$ spermidine, $1 \% \mathrm{BSA}, 10 \mathrm{mM}$ DTT, $40 \mathrm{U}$ RNasin, and $1 \mathrm{mM}$ of each ribonucleoside triphosphate), and the mixture was incubated for $2 \mathrm{~h}$ at $37{ }^{\circ} \mathrm{C}$. Transcribed RNA was stored at $-80{ }^{\circ} \mathrm{C}$ for a short time until use. Both linearised plasmid DNAs and transcribed infectious viral RNAs were quantified by agarose gel electrophoresis and spectrophotometry using a NanoDrop 1000 apparatus (Thermo Scientific).

\section{Production of virions}

HRV14 virions (wt and mutants) were produced in HeLa-H1 cells acquired from the ATCC. Cells were transfected by electroporation with non-mutated or mutant HRV14 viral RNA transcribed from pWR3.26-HRV14 plasmids as described above.

For comparing progeny virus production in quantitative assays, the same amount (15 $\mu \mathrm{g}$ in PBS) of wt and mutant viral RNAs was used for electroporation of the same number of cells in the same conditions. Electroporation of $5 \times 10^{6}$ cells were performed in a $0.5 \mathrm{ml}$ cuvette with one pulse at $980 \mathrm{~V}, 25 \mu \mathrm{F}$ and maximum resistance in a Gene Pulser II apparatus (BioRad). Control cells were electroporated in the absence of RNA but using the corresponding volume 
of PBS. Transfected cells were grown at $35^{\circ} \mathrm{C}$ in Petri dishes $(60 \mathrm{~mm}$ in diameter) during $4 \mathrm{~h}$ with $4 \mathrm{ml}$ of Dulbecco's Modified Eagle's medium (DMEM) containing glutamine and antibiotics, and supplemented with $10 \%$ fetal bovine serum (FBS), followed by prolonged incubation with the same medium supplemented with $2 \%$ FBS, until complete cytopathic effect was observed (usually $72 \mathrm{~h}$ post-transfection).

For preparation of large virus stocks, HeLa-H1 cell monolayers $\left(\sim 5 \times 10^{6}\right.$ cells in a $100 \mathrm{~mm}-$ diameter Petri dish), incubated until $\sim 90 \%$ confluency was reached, and infected with approximately $10^{7}$ plaque-formig units of HRV14 (either wt or mutants) during $1 \mathrm{~h}$ at $35^{\circ} \mathrm{C}$. After removal of the inoculum, $10 \mathrm{ml}$ of DMEM with glutamine and antibiotics, supplemented with $2 \% \mathrm{FBS}$, was added and the cells were incubated at $35{ }^{\circ} \mathrm{C}$ until complete cytopathic effect was observed (approximately 48-72 h post-infection). The supernatants of infection containing progeny viruses were collected, clarified by centrifugation, and used as described above to infect $\sim 90 \%$ confluent cell monolayers in $175 \mathrm{~cm}^{2}$ flasks (that had been seeded with $\sim 6 \times 10^{6}$ cells per flask). The supernatants containing amplified virus populations were collected, kept at $-80{ }^{\circ} \mathrm{C}$ and used for subsequent infections, or to proceed with virus purification.

\section{Purification of virions}

HRV14 virions (wt and mutants) were purified essentially as described, ${ }^{83}$ with minor modifications. Supernatants of infection containing viruses were frozen and thawed three times to disrupt remaining intact cells. Cell debris were removed by centrifugation. Then, the virus suspension on a $2 \mathrm{ml}$ sucrose cushion $(30 \% \mathrm{w} / \mathrm{v}$ sucrose, $1 \%$ sarcosyl, $0.1 \%$ mercaptoethanol in PBS) was centrifuged in a SW40 rotor (Beckman) at 40,000 rpm for 130 min at $16^{\circ} \mathrm{C}$. The pellet was thoroughly resuspended in PBS containing $0.01 \%$ bovine serum albumin (BSA), and the virus suspension was deposited on top of a 7.5-45\% sucrose gradient in PBS and centrifuged in the same rotor at $40000 \mathrm{rpm}$ for $110 \mathrm{~min}$ at $16^{\circ} \mathrm{C}$. 12-13 fractions of $1 \mathrm{ml}$ each were collected, and the presence of viral capsid protein in each fraction was analyzed by SDS-polyacrylamide gel electrophoresis. Fractions containing virions were pooled and thoroughly dialyzed against PBS. If needed, purified virion suspensions were concentrated by ultrafiltration (Amicon Ultracel 100K) at $4{ }^{\circ} \mathrm{C}$, and stored at $4{ }^{\circ} \mathrm{C}$ until use.

\section{Virus titration and inhibition of infectivity assays}

Infectious virus suspensions were titrated in standard plaque-formation assays. Briefly, nearly confluent HeLa-H1 cell monolayers in $60 \mathrm{~mm}$-diameter Petri dishes were inoculated with 200 
$\mu \mathrm{l}$ of serial dilutions of a virus suspension in PBS with $0.1 \%$ BSA. After incubation for $1 \mathrm{~h}$ at $35^{\circ} \mathrm{C}$, the inoculum was removed and cells were overlaid with a solid layer of $0.7 \%$ agar in DMEM supplemented with $1 \%$ FBS and $0.5 \%$ DEAE Dextran. The cells were then incubated at $35{ }^{\circ} \mathrm{C}$ for $72 \mathrm{~h}$, followed by staining with crystal violet. To ensure comparable results, a control non-mutated virus was included in each experiment. Titers were obtained at least in duplicate in each experiment, and the mean relative titers obtained for each mutant in independent experiments were averaged.

Antiviral drugs pleconaril (product code SML0307, Sigma-Aldrich) and pirodavir (product code R77975, MedChem Express) stocks were prepared by dissolving the compounds in $1 \%$ dimethyl sulfoxide (DMSO) in water at a concentration of $10 \mathrm{mg} / \mathrm{ml}$, and either used immediately or kept at $-80^{\circ} \mathrm{C}$ for a limited amount of time. Working stocks at 10 $\mu \mathrm{g} / \mathrm{ml}$ were prepared from the former stock by dilution in double distilled $\mathrm{H}_{2} \mathrm{O}$. Serial dilutions to be used in virus inhibition assays were prepared from the second stock in DMEM containing a final concentration of $0.001 \%$ DMSO.

Inhibition of virus infectivity by antiviral drugs was determined in infectivity assays essentially as previously described ${ }^{79}$ with some modifications, using purified virions that were preincubated for $2 \mathrm{~h}$ at $37{ }^{\circ} \mathrm{C}$ in the absence or presence of different concentrations of antiviral drug. Cells were seeded the day before the assay in 24-well tissue culture plates (at $1.25 \times 10^{5}$ cells per well). Near-confluent cell monolayers were infected at a multiplicity of infection (MOI) of 3. After incubation for $1 \mathrm{~h}$ at $35^{\circ} \mathrm{C}$ followed by removal of the inoculum, 1 $\mathrm{ml}$ of DMEM, supplemented with $2 \%$ FBS and the same concentration of antiviral drug used during the initial incubation of the virion suspension, and incubated for $18 \mathrm{~h}$ at $35^{\circ} \mathrm{C}$. Cell culture supernatants containing any progeny virions were then collected and titered as described above.

\section{Electron microscopy of virus particles}

For EM imaging of purified HRV14, undiluted samples were used. Virions were deposited on ionized Formvar/carbon-coated copper grids (Electron Microscopy Sciences), adsorbed for 4 min and washed three times with distilled water. Because HRV is acid-sensitive, a neutral negative staining agent, $2 \%$ sodium phosphotungstate at $\mathrm{pH} 7.2$, was used. Samples were stained for $45 \mathrm{~s}$, dried and visualized in a JEM-1010 electron microscope (JEOL). Images were taken with a TemCam-F416 camera (TVIPS). 


\section{Imaging and quantification of mechanical stiffness of virus particles by AFM}

Initial experiments were carried out to find the best conditions for high-resolution imaging and mechanical analysis. Purified virion samples were diluted into different buffers, including PBS and $50 \mathrm{mM}$ Tris- $\mathrm{HCl} \mathrm{pH}=7.4$ containing $5 \mathrm{mM} \mathrm{NiCl}_{2}$. Substrates included glass, silanized glass and freshly cleaved mica. Cantilevers used were RC800PSA (Olympus) with a nominal elastic constants $k_{c}=0.1 \mathrm{~N} / \mathrm{m}$ and $k_{c}=0.05 \mathrm{~N} / \mathrm{m}$. The final setup used in all AFM experiments involved silanized glass, PBS and cantilevers with $k_{c}=0.05 \mathrm{~N} / \mathrm{m}$. Imaging of isolated HRV14 virions by AFM was carried out essentially as described. ${ }^{15}$ The atomic force microscope (Nanotec Electrónica S.A.) was operated in liquid in Jumping Mode, ${ }^{66}$ controlled by WSxM software. ${ }^{84}$ At the beginning of each experiment, the actual spring constant of the cantilever in use was determined as previously described. ${ }^{85}$ Normal force during imaging was about $10-80 \mathrm{pN}$. AFM images were processed with WSxM software. ${ }^{84}$

Mechanical stiffness of wt and mutant HRV14 virions in the absence or presence of antiviral drugs was determined in indentation experiments using AFM, essentially as previously described. ${ }^{15}$ The instrumentation, cantilever, substrate and buffer were the same used for AFM imaging. To analyze the effect of virion-bound antiviral drugs on virion stiffness, purified virus suspensions diluted in buffer as needed were adsorbed onto silanized glass coverslips for $30 \mathrm{~min}$ at $25^{\circ} \mathrm{C}$. Then, the adsorbed virions were incubated for $30 \mathrm{~min}$ at $25^{\circ} \mathrm{C}$ with PBS solution in the absence or presence of drug at a saturating concentration (either pirodavir or pleconaril at $0.5 \mu \mathrm{M}$, diluted from a freshly prepared stock at $10 \mathrm{mg} / \mathrm{ml}$ in double distilled water plus 1\% DMSO). The elastic response of HRV14 virions were determined by obtaining F-Z curves well within the elastic regime, where forward and backward traces overlap and the maximal indentation was below $\sim 2 \mathrm{~nm}$, as determined by converting F-Z curves into F-D curves. ${ }^{15}$ Under these conditions, $k_{e}$ values that provide a measure of the stiffness of the viral particle were determined by considering sample and cantilever as two ideal springs in series, as previously described. ${ }^{15}$

\section{Statistical analysis of elastic constant values}

The $k_{e}$ values were fitted to a normal distribution described by a Gaussian fitting, as also observed for other virus particles. ${ }^{15,16}$ The statistical significance of $k_{e}$ differences was evaluated using OriginPro8 (OriginLab). To statistically validate that two $k_{e}$ distributions are different, the two population two-tailed Student t-test was used, with an alpha level $=0.05$. Equal variance was not assumed, providing a more stringent test. Statistical significance of 
differences between $k_{e} \mathrm{~s}$ of different viral particles and regions around different symmetry axes are respectively indicated in Tables S1 and S2 of SI.

\section{Molecular graphics and structural analyses}

The program $\mathrm{VMD}^{86}$ and the PDB atomic coordinates for the crystal structures of HRV14 virions, including wt (4RHV), wt with bound pleconaril (1NA1) and mutants V1188L (2RMU) and C1199Y (1RMU) were used to analyze structures, interpret results and/or display structural models. Contact analysis was performed using the program Whatif. ${ }^{87}$

\section{Conclusion}

The present study provides experimental support for the hypothesis that, from a fundamental physics point of view, pocket-binding antienteroviral drugs act by increasing virion stiffness (as determined by application of mechanical force). A quantitative relationship was observed between a linear increase in stiffness of the HRV14 virion by either binding antiviral drugs or introducing bulkier residues in capsid pockets, and a logarithmic reduction in its infectivity. This antiviral action may not be the consequence of a local rigidification around the pockets through direct steric effects. Rather, drug binding induces an overall, through quite subtle, rearrangement of the virion structure that greatly increases the stiffness of regions distant from the pockets. This lower overall deformability may translate into an increase in free energy difference between different conformations of the viral particle at equilibrium. In these conditions, thermal fluctuations at physiological temperature may no longer be able to readily promote the transition between these conformations, which would be observed as reduced virus breathing. As a consequence, receptor binding and genome uncoating are impaired, and virus infection is inhibited.

From an applied perspective, this study encourages the design of antiviral drugs that, as determined for enterovirus pocket-binding molecules, may inhibit infection by stiffening the virion. AFM-based quantification of stiffness, a basic material property of every virus and any other solid object, could be useful for initially assessing the potential of different virionstiffening compounds as antiviral drugs.

\section{Conflict of Interests}

There are no conflict of interests to declare. 


\section{Acknowledgements}

We thank Prof. Nuria Verdaguer for critical reading of the manuscript. A.V. was the recipient of a post-doctoral contract cofunded by Ministerio de Economía y Competitividad (MINECO) and Actuación Formación Postdoctoral 2013. M.G.M. is an associate member of the Institute for Biocomputation and Physics of Complex Systems, Zaragoza, Spain. This work was funded by grants to M.G.M. from MINECO/FEDER EU (BIO2012-37649 and BIO201569928-R) and by an institutional grant from Fundación Ramón Areces.

\section{References}

1. C. Bustamante, Z. Bryant and S.B. Smith, Nature, 2003, 421, 423-427.

2. C.M. Knobler and W.M. Gelbart, Annu.Rev.Phys.Chem., 2009, 60, 367-383.

3. W.M. Gelbart and C.M. Knobler, Science, 2009, 323, 11216-11221.

4. W.H. Roos, R. Bruinsma and G.J.L. Wuite, Nature Phys., 2010, 6, 733-743.

5. Emerging Topics in Physical Virology, eds. P.G. Stockley and R. Twarock, Imperial College Press, London, UK, 2010.

6. M.G. Mateu, Virus Res., 2012, 168, 1-22.

7. Structure and Physics of Viruses, ed. M.G. Mateu, Subcellular Biochemistry vol. 68, Springer, Dordrecht, The Netherlands, 2013.

8. P.J. de Pablo and M.G. Mateu, Subcell. Biochem., 2013, 68, 519-551.

9. A. Luque and D. Reguera, Subcell. Biochem., 2013, 68, 553-595.

10. E.R. May, Mol. Simul., 2014, 40, 878-888.

11. J.D. Perlmutter and M.F. Hagan, Annu. Rev. Phys. Chem., 2015, 66, 217-239.

12. M. Marchetti, G. Wuite and W.H. Roos, Curr. Opin. Virology, 2016, 18, 82-88.

13. U.F. Greber, J. Virol., 2016, 90, 3802-3805.

14. M.F. Hagan and R. Zandi, Curr. Opin. Virol., 2016, 18, 36-43.

15. I.L. Ivanovska, P.J. de Pablo, B. Ibarra, G. Sgalari, F.C. MacKintosh, J.L. Carrascosa, C.F. Schmidt and G.J.L. Wuite, Proc.Natl.Acad.Sci.USA, 2004, 101, 7600-7605.

16. C. Carrasco, A. Carreira, I.A.T. Schaap, P.A. Serena, J. Gómez-Herrero, M.G. Mateu and P.J. de Pablo, Proc. Natl. Acad. Sci. USA, 2006, 103, 13706-13711. 
17. J.P. Michel, I.L. Ivanovska, M.M. Gibbons, W.S. Klug, C.M. Knobler, G.J.L. Wuite and C.F. Schmidt, Proc. Natl. Acad. Sci. USA, 2006, 103, 6184-6189.

18. N. Kol, Y. Shi, M. Tsvitov, D. Barlam, R.Z. Schneck, M.S. Kay and I. Rousso, Biophys. $J ., 2007,92,1777-1783$.

19. C. Carrasco, M. Castellanos, P.J. de Pablo and M.G. Mateu, Proc.Natl.Acad.Sci.USA, 2008, 105, 4150-4155.

20. I.L. Ivanovska, R. Miranda, J.L. Carrascosa, G.J.Wuite, G.J. and C.F. Schmidt, Proc. Natl. Acad. Sci. USA, 2011, 108, 12611-12616.

21. M. Baclayon, G.K. Shoemaker, C. Uetrecht, S.E. Crawford, M.K. Estes, B.V. Prasad, A.J. Heck, G.J. Wuite and W.H. Roos, Nano Letters, 2011, 11, 4865-4969.

22. W.H. Roos, I. Gertsman, E.R. May, C.L. Brooks 3rd, J.E. Johnson and G.J. Wuite, Proc. Natl. Acad. Sci. USA, 2012, 109, 2342-2347.

23. M. Castellanos, R. Pérez, C. Carrasco, M. Hernando-Pérez, J. Gómez-Herrero, P.J. de Pablo and M.G. Mateu, Proc. Natl. Acad. Sci. USA, 2012, 109, 12028-12033.

24. H.B. Pang, L. Hevroni, N. Kol, D.M. Eckert, M. Tsvitov, M.S. Kay and I. Rousso, Retrovirology, 2013, 10, 4.

25. M. Hernando-Pérez, S. Lambert, E. Nakatani-Webster, C.E. Catalano and P.J. de Pablo, Nat. Commun., 2014, 5, 4520.

26. M. Hernando-Pérez, E. Pascual, M. Aznar, A. Ionel, J.R. Castón, A. Luque, J.L. Carrascosa, D. Reguera and P.J. de Pablo, Nanoscale, 2014, 6, 2702-2709.

27. S. Li, C. Sieben, K. Ludwig, C.T. Höfer, S. Chiantia, A. Hermann, F. Eghiaian and I.A.T. Schaap, Biophys. J., 2014, 106, 1447-1456.

28. R. Vaughan, B. Tragesser, P. Ni, X. Ma and B. Dragnea, Kao, C.C., J. Virol., 2014, 88, 6483-6491.

29. M. Castellanos, P.J.P. Carrillo and M.G. Mateu, Nanoscale, 2015, 7, 5654-5664.

30. A.Ortega-Esteban, G.N. Condezo, A.J. Pérez-Berná, M. Chillón, J. Flint, D. Reguera, C. San Martín and P.J. de Pablo, ACS Nano, 2015, 9, 10826-10833.

31. P.J.P. Carrillo, M. Medrano, A. Valbuena, Rodríguez-Huete, M. Castellanos, R. Pérez and M.G. Mateu, ACS Nano, 2017, 11, 2194-2208.

32. M. Cieplak and M.O. Robbins, J. Chem. Phys., 2010, 132, 015101.

33. O. Kononova, J. Snijder, M. Brasch, J. Cornelissen, R.I. Dima, K.A. Marx, G.J. Wuite, W.H. Roos and V. Barsegov, Biophys. J., 2013, 105, 1893-1903.

34. M. Cieplak, M and M.O. Robbins, PLoS One, 2013, 6, e63640. 
35. O. Kononova, J. Snijder, Y. Khodolov, K.A. Marx and V. Barsegov, PLoS Comput. Biol., 2016, 12, e1004729.

36. S.F. Cotmore and P. Tattersall, Annu. Rev. Virol., 2014, 1, 517-537.

37. The Picornaviruses, eds. E. Ehrenfeld, E. Domingo and R. Roos, ASM Press, Washington D.C, 2010.

38. M.G. Rossmann, E. Arnold, J.W. Erickson, E.A. Frankenberger, J.P. Griffith, H.-J. Hecht, J.E. Johnson, G. Kamer, M. Luo, A.G. Mosser, R.R. Rueckert, B. Sherry and G. Vriend, Nature, 1985, 317, 145-153.

39. S.E. Jacobs, D.M. Lamson, K. St. George and T.J. Walsh, Clin. Microbiol. Rev., 2013, 26, $135-162$

40. M.A. McKinlay, D.C. Pevear and M.G. Rossmann, Annu. Rev. Microbiol., 1992, 46, 635654.

41. M.G. Rossmann, Protein Sci., 1994, 3, 1712-1725.

42. J.M. Hogle, Annu. Rev. Microbiol., 2002, 56, 677-702.

43. C. Xiao, M.A. McKinlay and M.G. Rossmann, in Structural Virology, eds. M. AgbandjeMcKenna and R. McKenna, RSC Publishing, Cambridge, UK, 2011, ch. 3, pp. 319-337.

44. J.M. Casasnovas Subcell. Biochem., 2013, 68, 441-466.

45. D. Blaas, Wien Med. Wochenschr., 2016, 166, 211-226.

46. Y. Liu, M.G. Hill, T. Klose, Z. Chen, K. Waters, Y.A. Bochkov, W. Jiang, A.C. Palmenberg and M.G. Rossmann, Proc. Natl. Acad. Sci. USA, 2016, 113, 8997-9002.

47. S.R. Shih, S.J. Chen, G.H. Hakimelahi, H.J. Liu, C.T. Tseng and K.S. Shia, Med. Res. Rev., 2004, 24, 449-474.

48. A.M. de Palma, I. Vliegen, E. de Clercq and J. Neyts, Med. Res. Revs., 2008, 28, 823-884.

49. H.J. Thibaut, A.M. de Palma and J. Neyts, Biochem. Pharmacol., 2012, 83, 185-192.

50. F.G. Hayden, D.T. Herrington, T.L. Coats, K. Kim, E.C. Cooper, S.A. Villano, S. Liu, S. Hudson, D.C. Pevear, M. Collett, M. McKinlay, and the pleconaril Infection Study Group, Clin. Infect. Dis., 2003, 36, 1523-1532.

51. G.D. Diana, M.J. Otto and M.A. McKinlay, Pharmac. Ther., 1985, 29, 287-297.

52. T.J. Smith, M.J. Kremer, M. Luo, G. Vriend, E. Arnold, G. Kamer, M.G. Rossmann, M.A. McKinlay, G.D. Diana and M.J. Otto, Science, 1986, 233, 1286-1293.

53. J. Badger, I. Minor, M.J. Kremer, M.A. Oliveira, T.J. Smith, J.P. Griffith, D.M.A. Guerin, S. Krishnaswamy, M. Luo, M.G. Rossmann, M.A. McKinlay, G.D. Diana, F.J. Dutko, M. Francher, R.R. Rueckert and B.A. Heinz, Proc. Natl. Acad. Sci. USA, 1988, 85, 3304-3308. 54. M.G. Rossmann, Antiviral Res., 1989, 11, 3-14. 
55. Y. Zhang, A.A. Simpson, R.M. Ledford, C.M. Bator, S. Chakravarty, G.A. Skochko, T. Demenczuk, A. Watanyar, D.C. Pevear and M.G. Rossmann, J. Virol. 2004, 78, 11061 11069.

56. M. Roivainen, A. Närvänen, M. Korkolainen, M.L. Huhtala and T. Hovi, Virology, 1991, 180, 99-107.

57. Q. Li, A.G. Yafal, Y.M. Lee, J. Hogle and M. Chow, J. Virol., 1994, 68, 3965-3970.

58. J.K. Lewis, B. Bothner, T.J. Smith and G. Siuzdak, Proc. Natl. Acad. Sci. USA, 1998, 95, 6774-6778.

59. N. Reisdorph, J.J. Thomas, U. Katpally, E. Chase, K. Harris, G. Siuzdak and T.J. Smith, Virology, 2003, 314, 34-44.

60. L. Wang and D.L. Smith, Prot. Sci., 2005, 14, 1661-1672.

61. U. Katpally and T.J. Smith, J. Virol. 2007, 81, 6307-6315.

62. B. Bothner and J.K. Hilmer, in Structural Virology, eds. M. Agbandje-McKenna and R. McKenna, RSC Publishing, Cambridge, UK, 2011, ch. 3, pp. 41-61.

63. P.R. Kolatkar, J. Bella, N.H. Olson, C.M. Bator, T.S. Baker and M.G. Rossmann, EMBO $J ., 1999,18,6249-6259$.

64. F. Kienberger, A. Ebner, H.J. Gruber and P. Hinterdorfer, Acc.Chem.Res., 2006, 39, 2936.

65. A. McPherson and Y.G. Kuznetsov, Methods in Molecular Biology, 2011, 736, 171-195.

66. F. Moreno-Herrero, P.J. de Pablo, R. Fernández-Sánchez, J. Colchero, J. Gómez-Herrero and A. Baró, Appl.Phys.Lett., 2002, 81, 2620-2622.

67. M. Carrillo-Tripp, C.M. Shepherd, I.A. Borelli, S. Venkataraman, G. Lander, P. Natarajan, J.E. Johnson, C.L. Brooks 3rd and V.S. Reddy, Nucleic Acids Res., 2009, 37, D436-D442.

68. C. N. Hiremath, D.J. Filman, R.A. Grant and J.M. Hogle, Acta Cryst., 1997, D53, 558570 .

69. B.A. Heinz, R.R. Rueckert, D.A. Shepard, F.J. Dutko, M.A. McKinlay, M. Fancher, M.G. Rossmann, J. Badger and T.J. Smith, J. Virol., 1989, 63, 2476-2485.

70. J. Badger, S. Krishnaswamy, M.J. Kremer, M.A. Oliveira, M.G. Rossmann, B.A. Heinz, R.R. Rueckert, F.J. Dutko and M.A. McKinlay, J. Mol. Biol., 1989, 207, 163-174.

71. P. Guerra, A. Valbuena, J. Querol, C. Silva, M. Castellanos, A. Rodríguez-Huete, D. Garriga, M.G. Mateu and N. Verdaguer, Sci. Rep., 2017, 7, 4101.

72. B. Bothner, X. Fan Dong, L. Bibbs, J.E. Johnson and G. Siuzdak, J. Biol. Chem. 1998, 272, 673-676. 
73. B. Bothner, A. Schneemann, D. Marshall, V. Reddy, J.E. Johnson and G. Siuzdak, Nat. Struct. Biol., 1998, 6, 114-116.

74. R. Tuma, L.U. Coward, M.C. Kirk, S. Barnes and P.E. Prevelige Jr., J. Mol. Biol., 2001, 306, 389-396.

75. J.K. Hilmer, A. Zlotnick and B. Bothner, J. Mol. Biol., 2008, 375, 581-594.

76. C. Uetrecht and A.J.R. Heck, Angew. Chem. Int. Ed., 2011, 50, 8248-8262.

77. M. van de Waterbeemd, A. Llauró, J. Snijder, A. Valbuena, A. Rodríguez-Huete, M.A. Fuertes, P.J. de Pablo, M.G. Mateu and A.J.R. Heck, Biophys. J., 2017, 112, 1157-1165.

78. A. Roy and C.B. Post, Proc. Natl. Acad. Sci. USA, 2012, 109, 5271-5276.

79. K. Andries, B. Dewindt, J. Snoeks, R. Willebrords, K. van Eemeren, R. Stokbroekx and P.A.J. Janssen, Antimicrob. Agents. Chemother., 1992, 36, 100-107.

80. C. Lacroix, S. Laconi, F. Angius, A. Coluccia, R. Silvestri, R. Pompei, J. Neyts and P. Leyssen, Virol. J., 2015, 12, 106.

81. M. Bostina, H. Levy, D.J. Filman and J. Hogle, J. Virol., 2011, 85, 776-783.

82. D. Garriga, A. Pickl-Herk, D. Luque, J. Wruss, J.R. Castón, D. Blaas and N. Verdaguer, PLoS Path., 2012, 8, e1002473.

83. W. Wang, W.M. Lee, A.G. Mosser and R.R. Rueckert, J. Virol., 1998, 72, 1210-1218.

84. I. Horcas, R. Fernández, J.M. Gómez-Rodríguez, J. Colchero, J. Gómez-Herrero and A.M. Baró, Rev.Sci.Instrum., 2007, 78, 013705.

85. J.E. Sader, J.W.M. Chon and P. Mulvaney, Rev.Sci.Instrum., 1999, 70, 3967-3969.

86. W. Humphrey, A. Dalke and K. Schulten. J.Mol.Graph., 1996, 14, 33-38.

87. G. Vriend. J.Mol.Graph., 1990, 8, 52-56.

\section{Figure legends}

Figure 1. HRV14 structure. (A) high-resolution topographic model of the HRV14 virion obtained from the crystal structure ${ }^{38}$ (PDB ID: 4RHV). Average virion diameter is $\sim 30 \mathrm{~nm}$. Five-, three- and two-fold icosahedral symmetry axes are respectively labeled S5, S3 and S2. The virion surface is depth-cued, using colors from deep blue (lower radius) to yellow (higher radius). The canyon is seen as a deep blue convoluted groove around the yellow star-shaped mesa at each S5 axis. Entrance to each hydrophobic pocket underneath the canyon floor is highlighted in red. (B) close-up view of one of the 60 identical protomers in the HRV14 capsid represented as a cartoon model. Capsid proteins are colored blue (VP1), yellow (VP2), 
red (VP3) or black (VP4). The antiviral drug pleconaril bound inside the hydrophobic pocket $^{55}$ (PDB ID: 1NA1) is represented as a green spacefilling model. Distances between the pocket and S2 $(\sim 37 \AA)$ or S3 axes $(\sim 63 \AA)$ are indicated by black bars. (C) schematic lateral view of the hydrophobic pocket in the HRV14 capsid. Top, pocket filled with the antiviral drug pleconaril. Bottom, tested amino acid substitutions that introduce bulkier side chains in the pocket are shown.

Figure 2. Single HRV14 virions adsorbed to the substrate in different orientations. Left column: crystallographic atomic model of HRV virion ${ }^{38}$ (PDB ID: 4RHV) shown in three different orientations that present a different icosahedral symmetry axis (S2, S3 or S5) close to the top of the particle, color coded according to different heights in the particle along the viewing axis. Center left column: AFM images of three individual virions in PBS that present a different icosahedral symmetry axis (S2, S3 or S5) close to the top of the particle, color coded as for the atomic model. Horizontal scale bars are $15 \mathrm{~nm}$ in length. Center right column: filtered images of the viral particles imaged by AFM (center left) in which only an upper section is represented by a contour (black line) that corresponds to a same height. Right column: filtered images shown at the center right column were fed into a software that is able to identify polygons of different shapes. The polygons selected by the software were a rhombus (green), a triangle (blue) or a pentagon (red) for imaged particles that could be thus objectively assigned to different orientations with a S2, S3 or S5 axis on top, respectively (see text).

Figure 3. Individual HRV14 virions adsorbed on the substrate and $\boldsymbol{k}_{e}$ value distribution for the HRV14 virion. (A) AFM image of virions in PBS adsorbed to the substrate at low density. The horizontal scale bar is $200 \mathrm{~nm}$ in length. (B) the histogram represents the number of $F z$ curves determined that yielded $k_{e}$ values within each indicated range for adsorbed virions in PBS. The continuous line corresponds to a Gaussian fitting. See Table 1 for average $k_{e}$ value and standard deviation.

Figure 4. Comparison of elastic constants of different regions of the HRV14 virion in the absence or presence of drugs, and of HRV14 mutants. Bars indicate average $k_{e}$ values obtained for regions around S2 axes (red), S3 axes (green) or S5 axes (blue) for the nonmutated HRV14 virion (either in the absence of drug (wt) or presence of pirodavir (wtpiro) or pleconaril (wt-pleco)) and mutant virions V1188L, C1199Y and Y1152W. Standard 
deviations are indicated by thin bars. See Table 1 and Table S2 of SI for numerical $k_{e}$ values and standard deviations, and statistical analysis, respectively.

Figure 5. Anti-HRV14 activity of pocket-binding drugs. (A) pirodavir. (B) pleconaril. Equal amounts $\left(10^{5}\right.$ plaque infectious units) of HRV14 virions (wt, black circles, mutant C1199Y, brown diamonds, and mutant V1188L, green triangles) were incubated in the presence of the indicated drug concentrations before infecting susceptible cell monolayers. The progeny virus titers obtained (in plaque-forming units per $\mathrm{ml}$ ) are indicated as a function of drug concentration used.

Figure 6. Comparison of $k_{e}$ value distributions for the HRV14 virion in the absence or presence of pocket-binding antiviral drugs. Histograms represent number of events $(F-Z$ curves determined) that yielded $k_{e}$ values within each indicated range for HRV14 in the absence of any drug (No drug, top), or in the presence of a $0.5 \mu \mathrm{M}$ concentration of either pirodavir (center) or pleconaril (bottom) in PBS. Continuous lines correspond to Gaussian fittings. See Table 1 and Table S1 of SI for average $k_{e}$ values and standard deviations, and statistical analysis, respectively. Chemical structures of pirodavir and pleconaril are indicated.

\section{Figure 7. Structural and atomic mobility differences between crystallized HRV14} virions without or with bound pleconaril. Models of one of the 60 identical biological protomers of the HRV14 capsid are represented. (A) cartoon model of uncomplexed HRV14 (PDB ID: 4RHV); ${ }^{38}$ symmetry axes are indicated (pentagon for S5, triangle for S3, diamond for S2). (B) cartoon and (C) translucent spacefilling models of HRV14 with bound pleconaril (PDB ID: 1NA1). ${ }^{55}$ In (B) and (C), the orientation of the biological protomer and positions of the S5, S3 and S2 symmetry axes are the same depicted in (A), and the pleconaril molecule is depicted as a yellow spacefilling model. In (A) and (B), residues delimiting the capsid pocket that decreased their mobility, or that substantially changed their conformation and increased their mobility upon pleconaril binding, are respectively represented as red or green spacefilling models. In (B) and (C), all amino acid residues that decreased or increased their mobility when the drug was bound are respectively colored red or green. Changes in mobility

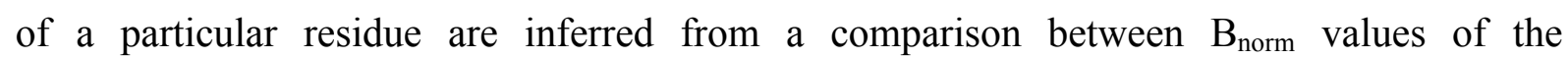
corresponding $\mathrm{C} \alpha$ in the HRV14 crystal structure without or with bound drug molecules (see text). In (C), the position of a capsid pocket is approximately delimited by a closed black line; 
the part of the protomer that participates in the interface between pentameric capsid subunits and that contains S2 and S3 axes is roughly indicated by an open black line.

Figure 8. Comparison of $k_{e}$ value distributions for the HRV14 wt and mutant virions Y1152W, C1199Y, V1188L. Histograms represent number of events ( $F-Z$ curves determined) that yielded $k_{e}$ values within each indicated range for HRV14 wt and mutants in PBS. Continuous lines correspond to Gaussian fittings. See Table 1 and Table S1 in SI for average values and standard deviations, and statistical analysis, respectively.

Figure 9. Plot of HRV14 infectivity versus average elastic constant of the virion. Virions tested included the wt virion either in the absence or presence of pirodavir or pleconaril mutants V1188A, V1188L, C1199Y and Y1152W with amino acid substitutions in the capsid pockets. Relative infectivity (drug-free nonmutated (WT) HRV = 1) is represented in a logarithmic scale. The data have been fitted to a logarithmic relationship indicated by a straight line in the semilogarithmic plot. 
Table 1. Elastic constant $\boldsymbol{k}_{\boldsymbol{e}}$ values $^{\mathrm{a}}$ for wt HRV14 in the absence or presence of pocketbinding drugs, and for HRV14 mutants

\begin{tabular}{|c|c|c|c|c|c|c|c|}
\hline $\begin{array}{c}\text { Virus } \\
\text { region } \\
\text { indented }^{\mathrm{b}}\end{array}$ & WT-drug ${ }^{c}$ & $\mathbf{W T}+\mathbf{p i r o}^{\mathrm{c}}$ & $\mathbf{W T}+$ pleco $^{\mathrm{c}}$ & V1188L & C1199Y & V1188A & Y1152W \\
\hline Average & $\begin{array}{c}0.49 \pm 0.20 \\
(52 / 15)\end{array}$ & $\begin{array}{c}0.86 \pm 0.43 \\
(72 / 21)\end{array}$ & $\begin{array}{c}0.85 \pm 0.41 \\
(55 / 11)\end{array}$ & $\begin{array}{c}0.49 \pm 0.23 \\
(71 / 14)\end{array}$ & $\begin{array}{c}0.58 \pm 0.24 \\
(50 / 9)\end{array}$ & $\begin{array}{c}0.49 \pm 0.28 \\
(10 / 2)\end{array}$ & $\begin{array}{c}0.83 \pm 0.30 \\
(43 / 8)\end{array}$ \\
\hline S2 & $\begin{array}{c}0.56 \pm 0.25 \\
(14 / 4)\end{array}$ & $\begin{array}{c}0.96 \pm 0.43 \\
(34 / 8)\end{array}$ & $\begin{array}{c}0.95 \pm 0.44 \\
(32 / 6)\end{array}$ & $\begin{array}{c}0.55 \pm 0.23 \\
(26 / 5)\end{array}$ & $\begin{array}{c}0.53 \pm 0.18 \\
(15 / 2)\end{array}$ & n.d. & $\begin{array}{c}0.81 \pm 0.37 \\
(25 / 5)\end{array}$ \\
\hline S3 & $\begin{array}{c}0.47 \pm 0.21 \\
(22 / 7)\end{array}$ & $\begin{array}{c}1.02 \pm 0.50 \\
(17 / 8)\end{array}$ & $\begin{array}{c}0.80 \pm 0.31 \\
(17 / 3)\end{array}$ & $\begin{array}{c}0.42 \pm 0.20 \\
(25 / 5)\end{array}$ & $\begin{array}{c}0.67 \pm 0.31 \\
(21 / 3)\end{array}$ & n.d. & $\begin{array}{c}0.89 \pm 0.16 \\
(16 / 2)\end{array}$ \\
\hline S5 & $\begin{array}{c}0.49 \pm 0.14 \\
(19 / 5)\end{array}$ & $\begin{array}{c}0.57 \pm 0.17 \\
(21 / 5)\end{array}$ & $\begin{array}{c}0.44 \pm 0.17 \\
(6 / 2)\end{array}$ & $\begin{array}{c}0.52 \pm 0.23 \\
(20 / 4)\end{array}$ & $\begin{array}{c}0.51 \pm 0.15 \\
(15 / 4)\end{array}$ & n.d. & $\begin{array}{c}0.68 \pm 0.18 \\
(2 / 1)\end{array}$ \\
\hline
\end{tabular}

${ }^{\mathrm{a}}$ Virions in PBS buffer at $25^{\circ} \mathrm{C}$. Values are the average \pm standard deviation of all individual measurements (indicated in parenthesis as number of $F-Z$ curves/number of particles indented). n.d., not determined

${ }^{\mathrm{b}}$ Average: average values obtained for all particles analyzed irrespective of orientation; S2, S3, S5: values obtained for particles oriented with a S2, S3 or S5 symmetry axis on top.

${ }^{\mathrm{c}}$ WT-drug, drug-free, non mutated HRV14; WT+piro, WT+pleco, nonmutated HRV14 incubated in the presence of $0.5 \mu \mathrm{M}$ pirodavir or pleconaril, respectively. 